OPEN ACCESS

Edited by:

András Büki,

University of Pécs, Hungary

Reviewed by:

John K. Yue,

University of California, San Francisco,

United States

Eugene Golanov, Houston Methodist Hospital,

United States

*Correspondence:

Tatiana Ruiz

tatiana.ruiz@mail.mcgill.ca

Specialty section:

This article was submitted to Neurotrauma

a section of the journal

Frontiers in Neurology

Received: 06 February 2019 Accepted: 02 July 2019

Published: 18 July 2019

Citation:

Ruiz T, Baldwin AS, Spiegel DP Hess $R$ and Farivar $R$ (2019) Increased Noise in Cortico-Cortical Integration After Mild TBI Measured With the

Equivalent Noise Technique.

Front. Neurol. 10:767.

doi: 10.3389/fneur.2019.00767

\section{Increased Noise in Cortico-Cortical Integration After Mild TBI Measured With the Equivalent Noise Technique}

\author{
Tatiana Ruiz ${ }^{1 *}$, Alex S. Baldwin ${ }^{1}$, Daniel P. Spiegel ${ }^{2}$, Robert Hess ${ }^{1}$ and Reza Farivar ${ }^{1}$ \\ ${ }^{1}$ Research Institute of the McGill University Health Center, Montreal, QC, Canada, ${ }^{2}$ Vision Sciences, Essilor R\&D, Center for \\ Innovation and Technology, Singapore, Singapore
}

The bulk of deficits accompanying mild traumatic brain injury (mTBI) is understood in terms of cortical integration-mnemonic, attentional, and cognitive disturbances are believed to involve integrative action across brain regions. Independent of integrative disturbances, mTBI may increase cortical noise, and this has not been previously considered. High-level integrative deficits are exceedingly difficult to measure and model, motivating us to utilize a tightly-controlled task within an established quantitative model to separately estimate internal noise and integration efficiency. First, we utilized a contour integration task modeled as a cortical-integration process involving multiple adjacent cortical columns in early visual areas. Second, we estimated internal noise and integration efficiency using the linear amplifier model (LAM). Fifty-seven mTBI patients and 24 normal controls performed a 4AFC task where they had to identify a valid contour amongst three invalid contours. Thresholds for contour amplitude were measured adaptively across three levels of added external orientation noise. Using the LAM, we found that mTBI increased internal noise without affecting integration efficiency. mTBI also caused hemifield bias differences, and efficiency was related to a change of visual habits. Using a controlled task reflecting cortical integration within the equivalent noise framework empowered us to detect increased computational noise that may be at the heart of mTBI deficits. Our approach is highly sensitive and translatable to rehabilitative efforts for the $\mathrm{mTBI}$ population, while also implicating a novel hypothesis of $\mathrm{mTBI}$ effects on basic visual processing - namely that cortical integration is maintained at the cost of increased internal noise.

Keywords: traumatic brain injury, internal noise, efficiency, contour perception, equivalent noise method, cortical integration

\section{SIGNIFICANCE STATEMENT}

Traumatic brain injury symptoms are largely understood in terms of neuronal and axonal loss, reflected in deficits that are largely understood in terms of cortical integration. An untested idea is that integration is maintained and compensated, but that injury causes increased computational noise. We tested this hypothesis using a psychophysical task with a strong neurophysiological basis that requires cortical integration and utilized an established approach to separately estimate internal noise and integration efficiency. Our results demonstrate that injury increases noise in cortical circuits without affecting integration efficiency. This sensitive and informed approach has important implications for diagnosis and rehabilitation of the two million U.S. patients affected annually by traumatic brain injury. 


\section{INTRODUCTION}

Traumatic brain injury (TBI) affects over 2,000,000 people in North America every year, with a sizeable portion of patients continuing to report deficits of attentional, mnemonic, or sensory nature many months after injury (1-5). The cognitive deficits of mild TBI (mTBI) can be present across different types or modes of injury, suggesting them to be general in nature (6). These deficits are often interpreted as a decreased capability of the cortical system to integrate information after injury.

Loss of tissue could have two distinct effects on the performance of a system-it could impair the integrative capacity of the system by reducing the efficiency with which information is processed, or it could increase the internal noise of the system. Here we aim to assess whether cortical changes caused by mTBI increase noise or decrease integration, or both.

Of the domains potentially affected by mTBI, the cortical visual system is the most characterized and best understoodthe human visual system has high homology to multiple animal models, and over 50 years of neurophysiology and psychophysics make it the most characterized cortical system $(7,8)$. Visual complaints are common after mTBI (9), and we and others have successfully used visual psychophysics to quantify cortical visual deficits caused by mTBI (10-14). The availability of highly sensitive psychophysical methods with physiologically-motivated computational models behind them make vision an excellent platform for characterizing and understanding cortical changes that follow mTBI.

High-level deficits such as memory and attentional losses can be broadly described as impairments of cortical integration over large cortical scales (15-17). A highly controllable model of cortical integration is contour integration-the perception of a shape through pooling of local edge segments that together describe a shape $(18,19)$. Contour integration is a crucial step in the processing of visual shape representation and is understood to require well-characterized integrative mechanisms at the lowest levels of the cortical visual hierarchy (20). Recently, a new contour integration approach has been developed that has the capability to allow measurements of both efficiency and internal noise (21) something not attainable from the original approach of Field et al. (18).

We therefore measured cortical integrative capacity and noise using the tightly-controlled visual contour integration task (21). Importantly, we can quantify both the capability of the cortical integration process that occurs for contours, as well as the amount of "noise" that is limiting the system's performance. To enhance our sensitivity to changes in integrative capacity and/or internal noise, we made our measurements independently for the four visual quadrants, which simultaneously enabled us to probe previously-reported visual field biases in $\mathrm{mTBI}(12,22)$.

\section{MATERIALS AND METHODS}

\section{Participants}

All participants gave their informed consent prior to taking part in the experiment. All procedures were in accordance with the Code of Ethics of the World Medical Association (Declaration of
Helsinki) and were approved by the Research Ethics Board of the McGill University Health Center.

All participants were screened for anomalous vision loss or vision disorders (glaucoma, retinal detachment, macular degeneration, etc.). They had normal or corrected to normal visual acuity (wore their usual refractive correction). The average age of the participants was 39.7 years old ( $\mathrm{SD}=14.4$ years, $n=$ $56)$ in the mTBI group and 35.5 years old $(\mathrm{SD}=13.8$ years, $n=$ 24 ) in the control group.

\section{TBI Group}

Participants (Table 1) were recruited through the McGill University Health Center out-patient TBI clinic. The diagnostic criteria for mild TBI were: Glasgow Coma Scale score between 13 and $15,<30 \mathrm{~min}$ of loss of consciousness, and $<24 \mathrm{~h}$ of amnesia regarding events immediately before or after the accident. Patients with mild TBI who gave their authorization to be contacted went through a phone screening interview. The exclusion criteria were (1) family history of epilepsy or seizure, or the administration of prescription medication with increased risk of seizure, (2) severe tremors or involuntary movements, (3) general anesthesia in the past 6 months, (4) mTBI occurred $<1$ month ago or more than 2 years ago, (5) presence of a brain lesion, (6) a history of multiple brain injury. During validation of patient's clinical history, we found that five of them had had previous head traumas, with their last one being a mild TBI (GSC 13-15). We removed these five subjects from our analysis, but their data were not discarded and instead, we analyzed them separately. We did not exclude participants on the basis of having received an intervention or not. Following our previous publication, participants filled a questionnaire adapted from Assessment with Mild Traumatic Brain Injury for the Defense Centers of Excellence for Psychological Health and Traumatic Brain Injury (13) investigating blurred vision, migraines, behavioral change to palliate visual discomfort etc. The final sample size of tested mTBI participants was 55 (13 males and 42 females), with an additional five polytrauma participants (two males, three females).

\section{Control Group}

Healthy participants were recruited through public announcements in the Montreal General Hospital and on social media. Demographics of the mTBI sample population were evaluated and the control group was sampled accordingly. Exclusion criteria included conditions 1-4 outlined above, and no history of any acquired brain injury. The control group was comprised of 23 individuals (12 males and 11 females). Despite the unequal proportions of males and females in both groups, sex had no effect on any of the LAM parameters, neither when taken as an average nor when assessed individually per quadrant $(p>0.05)$ and was therefore ruled out as a potential extraneous variable.

\section{Supplementary Evaluation}

The Trail Making Test B (23), the Bells Test (24), and the clock-drawing test (25) were administered to mTBI participants to assess visual attention and spatial neglect. All participants 
TABLE 1 | Participants.

\begin{tabular}{|c|c|c|c|c|c|c|c|c|c|c|}
\hline Subject & Age & Gender & TMT time & $\begin{array}{l}\text { TMT } \\
\text { errors }\end{array}$ & $\begin{array}{l}\text { Bells } \\
\text { time }\end{array}$ & Bells missed & $\begin{array}{l}\text { Education } \\
\text { Level }\end{array}$ & Handedness & Diagmsis & $\begin{array}{l}\text { Loss of } \\
\text { consciousness }\end{array}$ \\
\hline $\mathrm{t} 1$ & 59 & M & 29.699 & 0 & 66.38 & 5 & 11th Grade & Right & Mild complex & Yes \\
\hline t3 & 57 & M & 111.16 & 0 & 116.11 & 6 & Bachelor's Degree & Right & Mild & Yes \\
\hline t4 & 33 & M & 23.107 & 0 & 89.576 & 10 & Bachelor's Degree & Right & Mild simple & Yes \\
\hline $\mathrm{t} 7$ & 54 & $\mathrm{~F}$ & 25.989 & 0 & 115.183 & 1 & Bachelor's Degree & Right & Mild & Yes \\
\hline t8 & 40 & M & 16.01 & 0 & 65.93 & 0 & Bachelor's Degree & Right & Mild & No \\
\hline t9 & 64 & M & 24.6 & 0 & 80.29 & 5 & 11th Grade & Left & Mild & No \\
\hline $\mathrm{t} 10$ & 38 & $\mathrm{~F}$ & 38.2 & 0 & 115.1 & 2 & 11th Grade & Right & Mild & No \\
\hline $\mathrm{t} 11$ & 38 & $F$ & 33.646 & 0 & 82.928 & 6 & 11th Grade & Right & Mild simple & Yes \\
\hline $\mathrm{t} 16$ & 55 & $\mathrm{~F}$ & 37.65 & 0 & 117.786 & 2 & Bachelor's Degree & Right & Mild simple & No \\
\hline $\mathrm{t} 17$ & 53 & $\mathrm{~F}$ & 22.019 & 0 & 77.569 & 4 & Bachelor's Degree & Right & Mild trivial & No \\
\hline $\mathrm{t} 18$ & 32 & $\mathrm{~F}$ & 26.398 & 0 & 107.426 & 5 & Doctoral Degree & Right & Mild simple & No \\
\hline $\mathrm{t} 19$ & 41 & $\mathrm{~F}$ & 15.442 & 0 & 74.9 & 1 & Bachelor's Degree & Right & Mild & Yes \\
\hline $\mathrm{t} 20$ & 18 & $\mathrm{~F}$ & 25.933 & 0 & 76.599 & 3 & 11th Grade & Right & Mild simple & Yes \\
\hline $\mathrm{t} 21$ & 50 & $\mathrm{~F}$ & 23.369 & 0 & 68.446 & 6 & Professional DEC & Right & Mild simple & Yes \\
\hline $\mathrm{t} 22$ & 20 & $F$ & 27.62 & 0 & 50.909 & 13 & General DEC & Right & Mild simple & Yes \\
\hline $\mathrm{t} 23$ & 22 & $F$ & 14.8 & 0 & 60.5 & 14 & Bachelor's Degree & Left & Mild simple & No \\
\hline $\mathrm{t} 24$ & 46 & $\mathrm{~F}$ & 48.862 & 0 & 103.052 & 2 & Bachelor's Degree & Right & Mild simple & No \\
\hline $\mathrm{t} 25$ & 19 & $\mathrm{~F}$ & 19.98 & 0 & 46.6 & 6 & 11th Grade & Right & Mild complex & Yes \\
\hline t33 & 57 & $\mathrm{~F}$ & 27.33 & 0 & 75.3 & 7 & Bachelor's Degree & Left & Mild simple & Yes \\
\hline t34 & 32 & $\mathrm{~F}$ & 25.72 & 0 & 88.5 & 1 & Profession al DEC & Right & Mild simple & Yes \\
\hline t35 & 63 & $\mathrm{~F}$ & 22.14 & 0 & 89.19 & 1 & 11th Grade & Right & Mild complex & No \\
\hline t36 & 18 & $\mathrm{~F}$ & 23.11 & 1 & 101.84 & 1 & 11th Grade & Right & Mild simple & No \\
\hline t37 & 40 & M & 26.53 & 1 & 88.21 & 1 & Master's Degree & Right & Mild simple & Yes \\
\hline $\mathrm{t} 38$ & 23 & $\mathrm{~F}$ & 22.2 & 0 & 32.18 & 5 & Bachelor's Degree & Right & Mild simple & Yes \\
\hline t39 & 44 & $\mathrm{~F}$ & 19.93 & 1 & 62.08 & 11 & Bachelor's Degree & Right & Mild simple & No \\
\hline $\mathrm{t} 40$ & 24 & $\mathrm{~F}$ & 33 & 1 & 78.48 & 0 & Bachelor's Degree & Left & Mild simple & Yes \\
\hline $\mathrm{t} 41$ & 31 & $\mathrm{~F}$ & 23.28 & 0 & 119.65 & 2 & Bachelor's Degree & Right & Mild & Yes \\
\hline $\mathrm{t} 42$ & 28 & M & 32.35 & 1 & 65.43 & 7 & General DEC & Right & Mild complex & Yes \\
\hline t43 & 24 & M & 28.28 & 0 & 87.75 & 3 & Bachelor's Degree & Right & Mild simple & Yes \\
\hline $\mathrm{t} 44$ & 28 & $\mathrm{~F}$ & 20.55 & 0 & 46.37 & 7 & General DEC & Right & Self-reported & Yes \\
\hline $\mathrm{t} 45$ & 44 & M & 22.35 & 0 & 88.36 & 1 & Bachelor's Degree & Right & Mild simple & No \\
\hline $\mathrm{t} 46$ & 19 & $\mathrm{~F}$ & 13.28 & 0 & 78.84 & 2 & General DEC & Right & Self-reported & No \\
\hline $\mathrm{t} 47$ & 37 & $\mathrm{~F}$ & 22.53 & 0 & 62.68 & 2 & 11th Grade & Right & Mild & Yes \\
\hline $\mathrm{t} 48$ & 27 & $\mathrm{~F}$ & 31.26 & 0 & 122.4 & 1 & Bachelor's Degree & Right & Mild simple & Yes \\
\hline t49 & 24 & M & 35.49 & 1 & 183.07 & 0 & General DEC & Right & Mild simple & Yes \\
\hline
\end{tabular}

(Continued) 
TABLE 1 | Continued

\begin{tabular}{|c|c|c|c|c|c|c|c|c|c|c|}
\hline Subject & Age & Gender & TMT time & $\begin{array}{c}\text { TMT } \\
\text { errors }\end{array}$ & $\begin{array}{l}\text { Bells } \\
\text { time }\end{array}$ & Bells missed & $\begin{array}{l}\text { Education } \\
\text { Level }\end{array}$ & Handedness & Diagmsis & $\begin{array}{l}\text { Loss of } \\
\text { consciousness }\end{array}$ \\
\hline t50 & 45 & $F$ & 30.21 & 0 & 153.87 & 0 & General DEC & Right & Mild simple & No \\
\hline t51 & 53 & $\mathrm{M}$ & 35.63 & 1 & 138.45 & 0 & Master's Degree & Right & Mild simple & Yes \\
\hline t52 & 39 & $F$ & 24.58 & 0 & 61.93 & 2 & Bachelor's Degree & Right & Mild simple & No \\
\hline t53 & 50 & $F$ & 22.25 & 0 & 67.93 & 3 & Bachelor's Degree & Right & Mild simple & No \\
\hline t54 & 20 & $M$ & 16.8 & 1 & 77.47 & 3 & General DEC & Right & Mild simple & Yes \\
\hline t55 & 40 & $\mathrm{~F}$ & 43.1 & 0 & 171.8 & 1 & Bachelor's Degree & Right & Self-reported & $\mathrm{n} / \mathrm{a}$ \\
\hline poly1 & 24 & $\mathrm{M}$ & 24.5 & 0 & 122.57 & 1 & Bachelor's Degree & Right & Multiple & $\mathrm{n} / \mathrm{a}$ \\
\hline poly2 & 18 & $F$ & 14.3 & 0 & 40.74 & 4 & Master's Degree & Right & Multiple & $\mathrm{n} / \mathrm{a}$ \\
\hline poly3 & 26 & $\mathrm{~F}$ & 37.92 & 0 & 124.68 & 2 & $\mathrm{n} / \mathrm{a}$ & Right & Multiple & $\mathrm{n} / \mathrm{a}$ \\
\hline poly4 & 49 & $\mathrm{~F}$ & 36.34 & 0 & 98.31 & 10 & Bachelor's Degree & Right & Multiple & $\mathrm{n} / \mathrm{a}$ \\
\hline poly5 & 23 & $\mathrm{~F}$ & 33.44 & 0 & 110.39 & 1 & Bachelor's Degree & Right & Multiple & $\mathrm{n} / \mathrm{a}$ \\
\hline c1 & 42 & $F$ & $\mathrm{n} / \mathrm{a}$ & $\mathrm{n} / \mathrm{a}$ & 68.345 & 1 & Doctoral Degree & Right & None & $\mathrm{n} / \mathrm{a}$ \\
\hline c2 & 40 & $\mathrm{~F}$ & $\mathrm{n} / \mathrm{a}$ & $\mathrm{n} / \mathrm{a}$ & 76.398 & 4 & GEP General DE & Right & None & $\mathrm{n} / \mathrm{a}$ \\
\hline c3 & 53 & $\mathrm{~F}$ & $\mathrm{n} / \mathrm{a}$ & $\mathrm{n} / \mathrm{a}$ & 73.679 & 7 & Master's Degree & Left & None & $\mathrm{n} / \mathrm{a}$ \\
\hline c4 & 70 & $\mathrm{M}$ & $\mathrm{n} / \mathrm{a}$ & $\mathrm{n} / \mathrm{a}$ & 108.24 & 2 & Bachelor's Degree & Right & None & $\mathrm{n} / \mathrm{a}$ \\
\hline c5 & 19 & $\mathrm{M}$ & $\mathrm{n} / \mathrm{a}$ & $\mathrm{n} / \mathrm{a}$ & 68.66 & 1 & $\mathrm{n} / \mathrm{a}$ & $\mathrm{n} / \mathrm{a}$ & None & $\mathrm{n} / \mathrm{a}$ \\
\hline c6 & 54 & $\mathrm{M}$ & $\mathrm{n} / \mathrm{a}$ & $\mathrm{n} / \mathrm{a}$ & 92.8 & 1 & Master's Degree & Right & None & $\mathrm{n} / \mathrm{a}$ \\
\hline c7 & 49 & $\mathrm{~F}$ & $\mathrm{n} / \mathrm{a}$ & $\mathrm{n} / \mathrm{a}$ & 80.239 & 1 & EP Professional & Right & None & $\mathrm{n} / \mathrm{a}$ \\
\hline c8 & 18 & $\mathrm{~F}$ & 34.87 & 0 & 65.58 & 7 & 11th Grade & Right & None & $\mathrm{n} / \mathrm{a}$ \\
\hline c9 & 25 & $\mathrm{M}$ & 18.25 & 0 & 48.4 & 7 & Bachelor's Degree & Right & None & $\mathrm{n} / \mathrm{a}$ \\
\hline c10 & 21 & $\mathrm{~F}$ & 34.91 & 0 & 81.69 & 6 & Bachelor's Degree & Right & None & $\mathrm{n} / \mathrm{a}$ \\
\hline c11 & 53 & $\mathrm{~F}$ & 36.48 & 1 & & & Bachelor's Degree & Right & None & $\mathrm{n} / \mathrm{a}$ \\
\hline c12 & 42 & M & 12.92 & 0 & 57.43 & 1 & Bachelor's Degree & Right & None & $\mathrm{n} / \mathrm{a}$ \\
\hline c13 & 27 & $\mathrm{M}$ & 21.12 & 0 & 65.05 & 2 & Bachelor's Degree & Right & None & $\mathrm{n} / \mathrm{a}$ \\
\hline c14 & 50 & $\mathrm{~F}$ & 21.15 & 0 & 42.39 & 6 & $\mathrm{n} / \mathrm{a}$ & $\mathrm{n} / \mathrm{a}$ & None & $\mathrm{n} / \mathrm{a}$ \\
\hline c15 & 20 & $\mathrm{~F}$ & 28.32 & 0 & 56.76 & 1 & GEP General DE & Left & None & $\mathrm{n} / \mathrm{a}$ \\
\hline c16 & 26 & $\mathrm{M}$ & 21.54 & 0 & 46.37 & 5 & Mai.er1S Degree & Right & None & $\mathrm{n} / \mathrm{a}$ \\
\hline c17 & 35 & $\mathrm{~F}$ & 23.55 & 0 & 77.94 & 1 & Doctoral Degree & Right & None & $\mathrm{n} / \mathrm{a}$ \\
\hline c18 & 46 & M & 12.63 & 0 & 66.11 & 3 & Bachelor1s Degree & Right & None & $\mathrm{n} / \mathrm{a}$ \\
\hline c19 & 25 & $\mathrm{M}$ & 16.85 & 0 & 72.67 & 3 & 11th Grade & Right & None & $\mathrm{n} / \mathrm{a}$ \\
\hline c20 & 22 & $\mathrm{~F}$ & 19.1 & 1 & 687 & 0 & Bachelor's Degree & Right & None & $\mathrm{n} / \mathrm{a}$ \\
\hline c21 & 28 & $\mathrm{M}$ & 23.53 & 0 & 62.98 & 4 & Doctoral Degree & Right & None & $\mathrm{n} / \mathrm{a}$ \\
\hline c22 & 27 & M & 15.16 & 1 & 48.19 & 3 & Master's Degree & Right & None & $\mathrm{n} / \mathrm{a}$ \\
\hline c23 & 33 & $M$ & 32.72 & 0 & 126.14 & 0 & Doctoral Degree & Right & None & $\mathrm{n} / \mathrm{a}$ \\
\hline
\end{tabular}

responded normally on these tests. Monocular and binocular visual acuity was measured with a Snellen chart at four meters (Logarithmic Visual Acuity Chart; Precision Vision, Lasalle, IL, USA) and their ocular dominance was assessed using the Miles test. Maddox rod, cover/uncover and alternating-cover tests were performed to detect presence of strabismus. Participants were excluded from the study if a strabismus was found.

\section{Display}

Stimuli were produced using Psychtoolbox (26) through MATLAB $^{\circledR} \quad(2014 \mathrm{~b}$, The Math Works Inc., Natick, Massachusetts) (27) and presented on a gamma-calibrated LG Flatron 915FT Plus monitor using a 10-bit graphics card (Nvidia Quadro 2000). Calibration was done using a photometer, and the mean luminance was $62 \mathrm{~cd} / \mathrm{m}^{2}$. Subjects were placed consistently at a $77 \mathrm{~cm}$ viewing distance from the monitor, with a spatial resolution of 96 pixels per degree of visual angle.

\section{Stimuli and Procedure}

Subjects fixated on a marker at the center of the screen. On each trial, four contours appeared simultaneously. Each contour was centered in one quadrant of the visual field, at an eccentricity of $2.8^{\circ}$ from fixation. Each contour was comprised of seven logGabor wavelets (28) resting on an invisible curved path. The wavelets had a peak spatial frequency of $6 \mathrm{c} / \mathrm{deg}$ with a bandwidth of 1.6 octaves. They were presented in cosine phase (white bar with dark flanks) and had an orientation bandwidth of $\pm 25^{\circ}$. The full-width at half-magnitude of the wavelet envelopes measured 1.17 cycles along the stripes, and 0.91 cycles across them. For the target the orientation of each wavelet was aligned with the path of the contour. In the three distractors, the orientations of the 
A

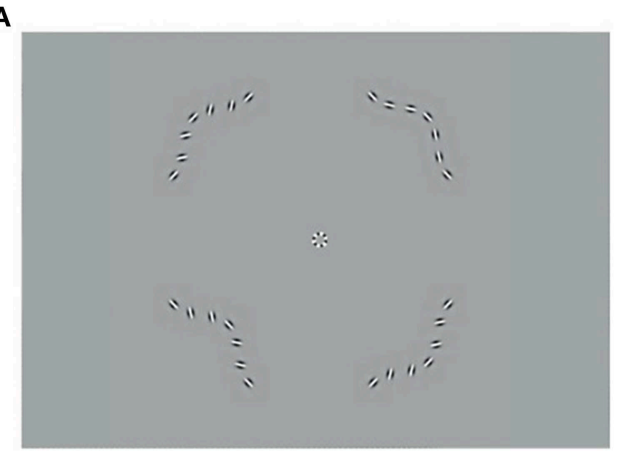

B

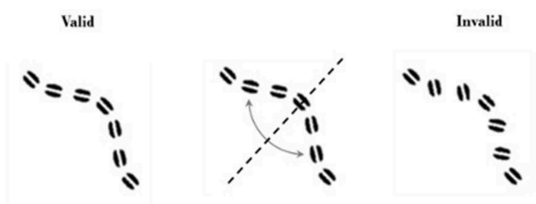

C

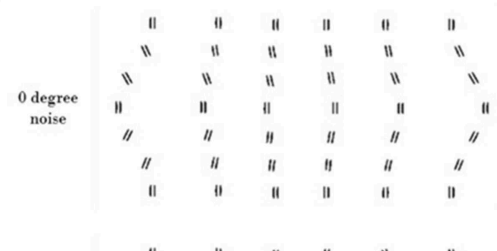

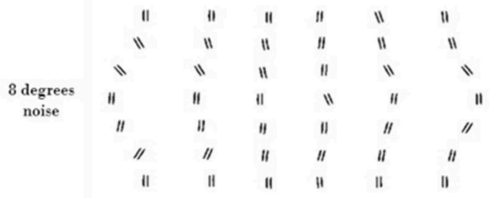

16 degrees "
noise "

FIGURE 1 | Visual stimuli. Contrast has been enhanced for illustration purposes. (A) An example trial of the Good continuation discrimination task, the upper right quadrant contains the valid contour $\left(0^{\circ}\right.$ noise). (B) Construction of the invalid contour by inverting elements across the valid contour diagonal. Because of the systematic nature of this process, it is not to be confused with the addition of orientation noise. (C) Cartoon showing valid and invalid contours varying in amplitude under noise levels 1,2 , and 3 (respectively $0^{\circ}, 8^{\circ}$, and $16^{\circ}$ of SD of orientation noise). Note the increasing difficulty of discriminating between the valid (on the right) and invalid (on the left) contours as amplitude decreases.

wavelets were consistent with a contour curving in the opposite direction. Discriminating the target from the distractors required the subject to combine the orientation and position information of the wavelets (Figure 1).

The contour paths that specified the wavelet locations had the same amplitude for the target and for the three distractors. For large curvature amplitudes the task is easier, as the target appears to be a smoother contour than the distractors. Stimuli were presented for $400 \mathrm{~ms}$, and subjects selected the smoother contour (with "good continuation") (29) in a four-alternate forced choice (4AFC) task (Figure 1). This task was chosen to ensure data could be collected efficiently from inexperienced subjects (30). The amplitude of the curvature was modulated through a performance-dependent staircase (2-down 1-up), converging at an amplitude where the subject selected the target $70 \%$ of the time. The staircase was terminated after 40 trials or following 12 reversals. Thresholds for identifying the smooth contour were obtained using psychometric function fitting (see below). Thresholds were obtained both for stimuli without any added external noise, and for stimuli where the orientations of the individual wavelets were randomly jittered. Measuring performance at different levels of external noise allows the equivalent internal noise and processing efficiency of the contour integration system to be characterized. This method has been previously validated, with human performance measurements quantified compared to that from the ideal observer (21).

We measured discrimination thresholds at three levels of orientation noise: $0^{\circ}, 8^{\circ}$, and $16^{\circ}$. The orientation of each wavelet was resampled from a Normal distribution centered on its original value (aligned with the contour for the target stimuli, and consistent with a contour of opposite curvature for the distractors). The standard deviation of the Normal distribution controlled the level of external orientation noise. We divided data collection into separate blocks for each noise level (10-15 min each). The order of these blocks was randomized across participants. We have created an interactive illustration of the procedure and corresponding psychometric performance hosted at http://www.farivarlab.com/ stimuli-software.

\section{Experimental Design and Statistical Analysis \\ Experimental Design}

We utilized a $2 \times 2 \times 2$ factorial design, with a between-subjects factor (mTBI vs. controls) and two within-subject factors of vertical visual field (upper vs. lower) and horizontal visual fields (left vs. right). To make inferences of differences in internal noise and efficiency across the subjects and quadrants, we analyzed these parameters as estimated by the Linear Amplifier Model (see below) using non-parametric tests (31). To make inferences about quadrant biases, we used the rank assignment of each quadrant for internal noise and efficiency and carried out the same non-parametric tests on these rank values.

\section{Statistical Analysis}

mTBI subjects tend to be heterogenous and their performance often does not follow a normal distribution-something we have previously observed $(13,14)$. Our data here also were not normally distributed, and we therefore carried out all our analyses using non-parametric inferential tests, which are more 


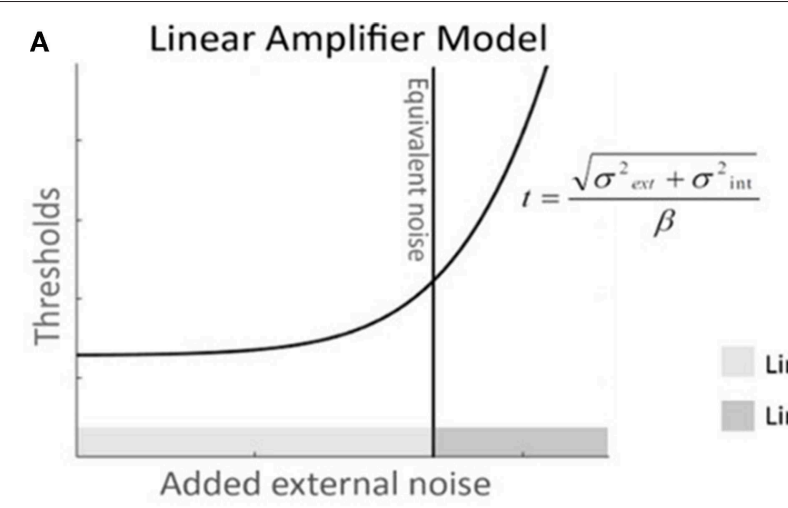

Limited by internal noise

Limited by external noise

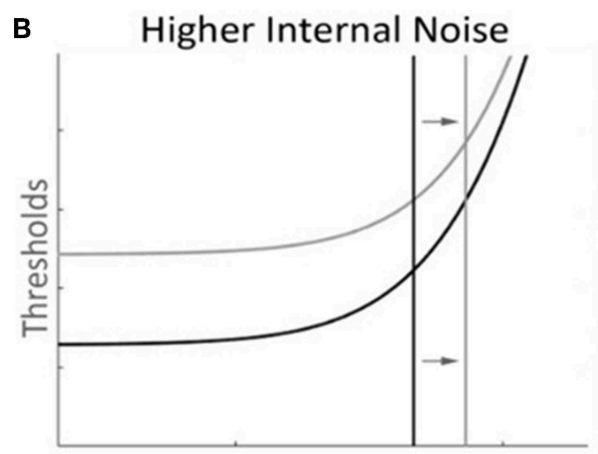

Added external noise

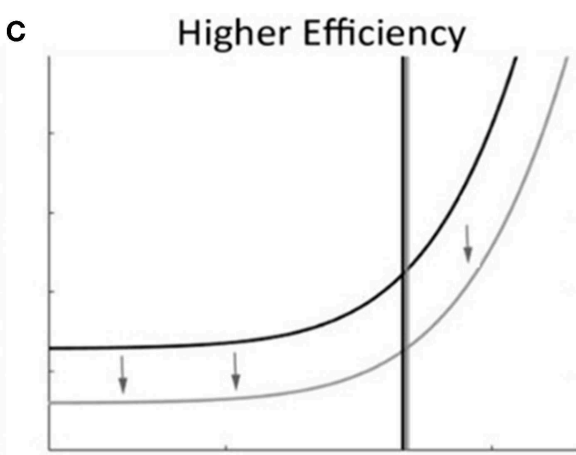

Added external noise

FIGURE 2 | Linear Amplifier model (LAM) graphical description with mock-data. (A) The LAM function describes the dynamics of performance thresholds along levels of added external noise - thresholds ( $t)$, as a function of external noise $\left(\sigma_{\text {ext }}\right)$, internal noise $\left(\sigma_{\text {int }}\right)$, and efficiency $(\beta)$. At low levels of external noise, performance is not dependant on external noise and remains constant and limited by internal noise. After the equivalent noise point, additional external noise shifts thresholds upwards, and becomes the major limiting factor of performance. (B) A higher internal noise curve (in gray) with unchanged efficiency shows a shift in the equivalent noise point toward higher noise. The thresholds are shifted up, as the tail of the function asymptotes toward the same slope. (C) A higher efficiency curve (in gray) with unchanged internal noise shows a global shift toward lower thresholds and maintains the same equivalent noise point.

conservative and do not depend on normality of the data distribution and here report the Wald-type statistic (WTS) estimated using the nparLD (31) package in the R Statistical Package (32), which is a non-parametric analog of the repeatedmeasures factorial ANOVA.

\section{Data Pre-processing and Psychometric Fitting}

Psychometric function fitting was performed using the Palamedes toolbox (33). The number of trials at each curvature amplitude, and the number of correct responses for each amplitude were fitted with a Gumbel psychometric function. The guessing rate parameter was fixed at 25\% (guessing rate for a 4 AFC task). The lapse rate was allowed to vary from 0 to $5 \%$, while the threshold and the slope were allowed to vary across noise levels and quadrants.

The equivalent noise method, borrowed from engineering (34-37), uses external noise added to the input of a system to measure the equivalent internal noise level within the system. When the external noise is much smaller than the equivalent internal noise then its effects will be negligible. As the external noise is increased it will reach a point where its effects exceed those of the equivalent internal noise. Beyond this point the external noise will dominate performance, making the system's equivalent internal noise no longer the limiting factor. Psychophysically, noise masking experiments typically find that thresholds are unaffected by low levels of external noise. Beyond some critical value however, the thresholds increase in proportion to the standard deviation of the masking noise. The simplest model for analyzing data from equivalent noise studies is the Linear Amplifier Model (LAM), which has two parameters

$$
A_{\text {threshold }}=\frac{\sqrt{\sigma_{\text {external }}^{2}+\sigma_{\text {internal }}^{2}}}{\beta} .
$$

This predicts a threshold $A_{\text {threshold }}$ for each external noise level $\sigma_{\text {external }}$. The fitted $\sigma_{\text {internal }}$ parameter indicates the point at which the system transitions from being dominated by internal noise to being dominated by external noise. This is taken as the external noise level that is equivalent to the internal noise level. The second fitted parameter $\beta$ indicates the processing efficiency of the system (38). Elevated internal noise will affect thresholds when the external noise is low or absent but will not change behavior once external noise is greater than internal 
noise. Reduced efficiency however will increase thresholds at all external noise levels. In the context of our contour task, internal noise indicates the inherent internal limitations affecting the representation of each wavelet, while the efficiency is the capability of the system to combine all of that noisy information to detect good continuity. Thus, the LAM model effectively captures the two key dimensions of performance that we aimed to measure.

The LAM was then fitted to the discrimination thresholds to determine internal noise and efficiency (Figure 2). Outlier participants were removed if one of their LAM parameters was further than 1.5 interquartile below Q1 or above Q3 for each group $(39,40)$, leaving 21 controls (two outliers) and $50 \mathrm{mTBI}$ subjects (five outliers).

Following data collection, we noted that the highest amplitude of curvature produced contours that were difficult to discriminate for several participants in both groups. This was true even at low noise levels. We designed an unbiased means of eliminating these points and validating that our procedure did not bias the results. The data points collected at these amplitudes were unreliable (they resulted in non-monotonic psychometric functions). We removed these data points if doing so significantly improved the fit of the Gumbel function to the data, as determined by a Chi-square goodness-of-fit test. Eleven control subjects and 20 mTBI subjects had points removed. Within-subject comparison of before and after the outlier removal showed no significant difference across groups for all quadrants and all noise levels which means that the group differences found after fitting the LAM were not biased by our outlier removal procedure (WTS $0.89 p>0.3)$. The unbiased preprocessing step significantly decreased variability across the pool of all participants across both groups for quadrants 1,2 and 3 (WTS $8.5 p=0.0035$, WTS $4.4 p=0.035$, WTS $9.57 p=0.002$ ) and for noise level 2 (WTS $6.67 p=0.01)$.

\section{RESULTS}

\section{Higher Internal Noise Following TBI}

Internal noise was significantly higher in the mTBI group than in the control group (Figures 3A,B) ( WTS $=8.64, p=0.003)$. We noted a significant interaction in the visual field biases between group and horizontal hemifields ( $W T S=7.97, p=0.005$ ). Control subjects had lower internal noise than mTBI subjects (in both horizontal hemifields) with even lower internal noise in the right hemifield than in the left.

Analyzing the data within groups, we found that control subjects presented a significant horizontal bias $(W T S=4.86$, $p=0.03$ ) with lower internal noise in the right hemifield as opposed to mTBIs who did not have any hemifield bias in internal noise (horizontal $W T S=2.78, p=0.09$, vertical $W T S=3.43$, $p=0.06$ ) (Figure 3C).

\section{Abnormal Efficiency Distribution Across the Visual Field Following TBI}

Although there was no group difference in efficiency overall ( $W T S=0.85, p=0.36$ ), efficiency remained constant across hemifields in the control group (all $p$ 's $>0.1$ ) whereas mTBI subjects presented significant horizontal and vertical biases (horizontal $W T S=12.52, p=0.0004$, vertical $W T S=$ 11.78, $p=0.0006$ ) with higher efficiency in the lower right quadrant (Figure 3D).

\section{Consistent Visual Field Quadrant Ranking After TBI}

To assess potential visual field imbalances caused by mTBI, for each subject we rank-ordered the quadrants in terms of internal noise and efficiency (separately) and analyzed these rank scores using the same non-parametric method described above. The mTBI group exhibited visual field biases as measured by rank of both efficiency and internal noise (efficiency WTS $=20, p=$ 0.0002 , internal noise $W T S=9.5, p=0.23$ ); this was not observed in the control group (efficiency WTS $=0.3, p=0.96$, internal noise $W T S=2.15, p=0.54$ ).

mTBI subjects presented significant horizontal and vertical biases $(W T S=9.9 p=0.0016 ; W T S=9.1 p=0.002$, respectively) in efficiency, and a significant vertical bias $(W T S=5.74 p=$ 0.017 ) in internal noise. Control participants presented none of these biases.

\section{Efficiency and Internal Noise Positively Correlated in Both Groups}

To understand the dynamic relationship between efficiency and internal noise, we tested for correlations between these parameters for each individual quadrant in each group and found a significantly positive correlation between internal noise and efficiency in all quadrants for both groups (Spearman, rho $>0.4, p<0.001)$. However, when looking at the parameters averaged across quadrants this positive correlation was only maintained in the mTBI group (Spearman, rho $=0.36, p=$ 0.01). All participants tended to have higher efficiency in the quadrants where they also exhibited higher internal noise, but only participants from the mTBI group compensated for higher internal noise with higher efficiency when all quadrants were taken into account.

\section{Internal Noise, Efficiency, and Visual Dysfunctions Reports}

To investigate the relationship between the internal noise and efficiency parameters as measured by our task and the symptoms experienced by the patients, we tested whether their answers to the Visual symptoms questionnaires were correlated to their internal noise and efficiency. Interestingly, changes in visual habits were inversely correlated to the efficiency on the goodcontinuity discrimination task (Spearman, rho $=-041, p=$ 0.04), meaning that the more patients made changes to their visual habits (screen time, reading, driving...) the less efficiency they exhibited at discriminating between valid and invalid contours. Although the strength of the correlation was small, this would suggest that patients who adapted their behavior to their visual impairments also showed less efficiency in using the available orientation information to render a perceptual decision. 

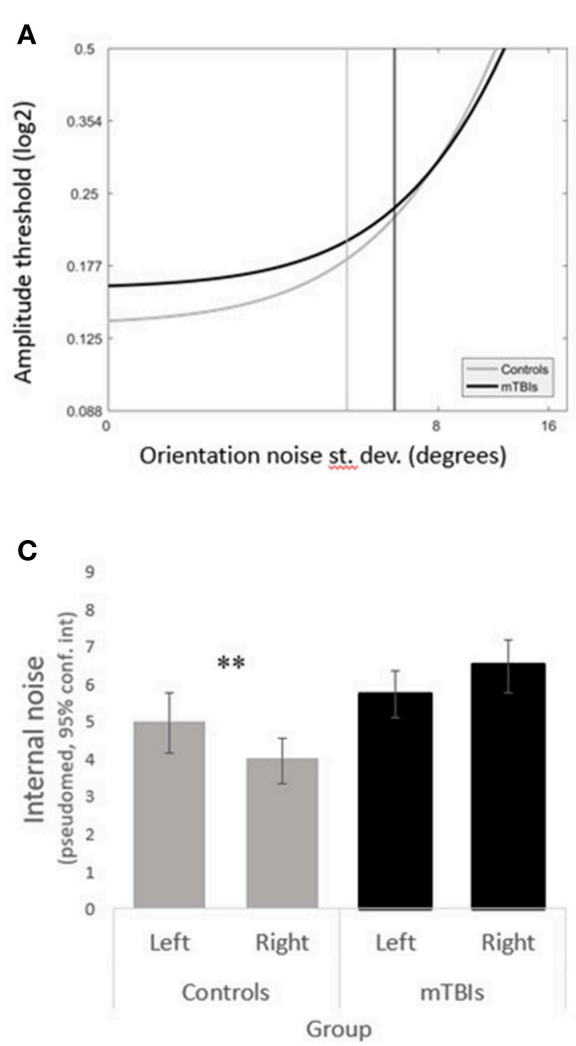

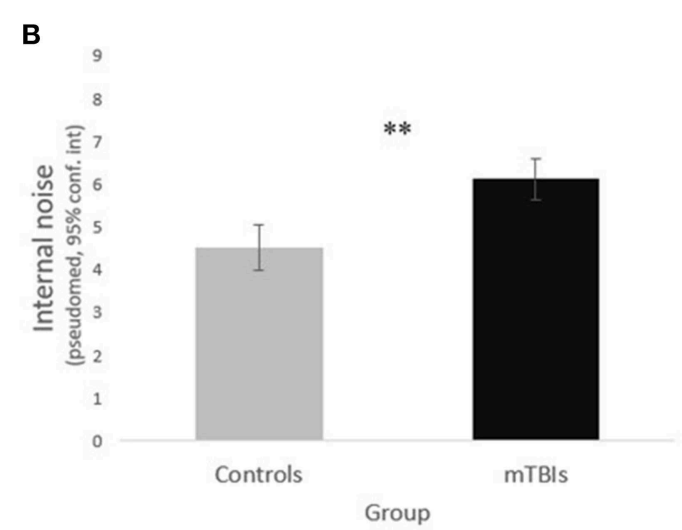

D

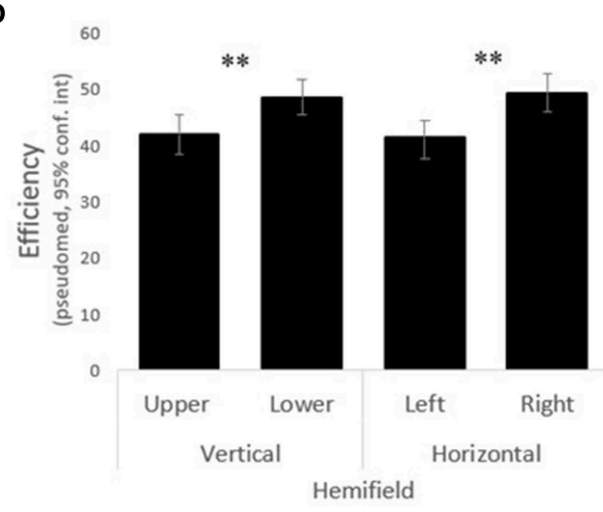

FIGURE 3 | Main results. (A) LAM functions for the mTBI subjects (in black) and the control subjects (in gray). (B) The mTBI group shows significantly higher internal noise than the control group. (C) The left and right hemifields varried significantly in internal noise in the control group but not in the mTBI group. (D) mTBI subjects had significant biases in efficiency across both the vertical and horiontal hemifields.

\section{Time Since Injury}

Studies looking at post-concussive symptoms typically span their data collection between the time of injury and the following year, finding a decrease when comparing time points (41-43). Surprisingly, when we tested whether internal noise or efficiency on the good-continuity discrimination task were correlated with the time elapsed since injury, we did not find any significant relationship (Spearman, rho $<-0.1, p>0.4$ ). We did not find any relationship between the time since injury and any of the neuropsychology tests either.

\section{Multiple Concussion Participants}

For the five multiple concussion participants that were tested on the contour discrimination task, internal noise was marginally higher than in the controls (WTS $=3.76 p=0.053$ ), and not different from the single mTBI group (WTS $=0.27 p>0.6$ ). When analyzing all three groups at once (Table 2), we found a significant main effect of Group on internal noise (WTS $=$ $9.9 p<0.007$ ), as well as a significant interaction between the factors Group and Quadrants (WTS $=37.8 p<0.000001$ ). The multiple TBI group had higher internal noise (pseudo-median $=$ $6.69^{\circ}$, conf.int $=5.29^{\circ}-8.56^{\circ}$ ) than the single TBI group (pseudomedian $=6.1^{\circ}$, conf.int $=5.65^{\circ}-6.58^{\circ}$ ), and the control group
TABLE 2 | Result summary.

\begin{tabular}{ll}
\hline Single mTBI & Polytrauma \\
\hline $\begin{array}{l}\text { Higher internal noise than } \\
\text { controls }\end{array}$ & $\begin{array}{l}\text { Higher internal noise than controls and } \\
\text { single mTBls }\end{array}$ \\
$\begin{array}{l}\text { Abnormal efficiency distribution } \\
\text { across visual field }\end{array}$ & No effect found on efficiency
\end{tabular}

had even lower internal noise (pseudo-median $=4.51^{\circ}$, conf.int $=3.99^{\circ}-5.06^{\circ}$ ).

Efficiency of the multiple TBI patients did not vary compared to either group separately (controls/multiple TBIs $W T S=1.12$ $p>0.2$, TBIs/multiple TBIs WTS $=0.45 p>0.4$ ). When the data from the three groups were combined into a single analysis, neither Group nor Quadrants had a significant relative effect on efficiency $(W T S=1.5 p=0.48 ;$ WTS $=2.2 p=0.53$, respectively), but there was an effect of the interaction of Group and Quadrants on efficiency ( $W T S=20.8 p=0.002$ ).

\section{DISCUSSION}

Cortical integration is understood to be at the heart of many cognitive symptoms related to attention and memory following mTBI-a large network of cortical regions is engaged to carry 
out these fundamental cognitive processes $(15,16,44)$. Directly measuring impairments of cortical integration is a serious challenge, because of the absence of informed quantitative models that fully capture the two crucial limiting factors, namely cortical integration and internal noise. By utilizing contour integration - a fundamental step in visual shape recognition that is well-characterized in terms of cortical integration $(20,45)-$ within the framework of the equivalent noise technique, we were able to overcome the limitations posed by cognitive measures while assessing changes in visual processing following mTBI.

We have discovered that mTBI may not actually result in less efficient cortical integration per se, but rather in increased internal noise. This is a first quantitative characterization of the post-TBI changes using a model-driven behavioral task (46-50). Our results also corroborated the previous finding of visual field biases being affected by $\mathrm{mTBI}$ - we found that cortical integration efficiency was different between the vertical and horizontal hemifields. Finally, we found that poorer cortical integration efficiency was correlated with greater change in visual habits of mTBI patients.

We observed a significant increase in internal noise despite the recognized variability in the mTBI population $(51,52)$, suggesting that internal noise is a valuable and valid construct in describing the visual processing changes that occur in this disorder. Occipital injury was not a common mode of insult, yet the bulk of the group exhibited elevated internal noise on a visual task. Adding to the emerging scientific evidence for cortical visual impairments following mTBI $(11-14,22)$, our experiment relied on a non-invasive psychophysical method to probe cortical errors and inefficiencies in the low- to mid-level visual areas of the human brain $(20,45,53)$.

\section{Cortical Integration During Contour Perception}

Contour integration is a basic building block of visual perception, and yet, it requires complex and balanced interactions $(18,19$, $54,55)$. This integrative process can be effectively probed using simplified stimuli consisting of colinear Gabor elements along a path defining a shape-in our case, a simple arc. Such colinear sets tend to pop-out against a background of randomly-oriented Gabors, as captured by the Gestalt rule of Good Continuation $(29,56,57)$. Thus Good Continuation is the fundamental feature of a contour perception, and the task used here (21) directly measures this key aspect of visual perception.

The perception of a contour is not instantaneous $(19,58,59)$ suggesting multiple levels of computation, and recent evidence suggests at least two major steps are involved-a first step where the individual elements are detected by V1 neurons and a second step where secondary connections (lateral in V1 and/or feedback from extrastriate areas) "fill-in" the gaps between the Gabor elements (20). In other words, the individual Gabor elements of a synthetic contour each have distinct cortical representations in the retinotopic map of V1 (55). These individual cortical representations then interact and integrate into a new form-the full contour-thus describing a simple and elegant example of cortical integration that can be tightly controlled via stimulus manipulations.
Although there are diverging views regarding the cortical mechanisms involved in contour perception, namely if linking between stimulus elements is explicit or not $(18,60,61)$, some form of integration remains unavoidable, whether it follows a step-by-step summation or an algorithmic overlap of orientation and template filters across hierarchical processing levels. We propose that contour integration can serve as an effective model of cortical integration, because the individual elements of a contour have distinct cortical representations and because the integration of the contour requires pooling and interactions across a set of such cortical nodes. The magnitude of these interactions can be controlled by stimulus parameters such as collinearity, gap, and path curvature (62-65), unlike cortical interactions engaged in complex cognitive tasks. Given the tight control that is granted by stimulus manipulations on this well-characterized integrative cortical process, contour integration is an effective and efficient method of probing complex interactions in the injured brain.

\section{Cortical Visual Deficits After TBI}

We had previously speculated that long-range fibers-i.e., those that integrate information across visual fields and cross at the corpus callosum-are most vulnerable to injury in mTBI (12). We and others $(12-14,66)$ have documented several changes to cortically-mediated visual processes after mTBI. Traumatic brain injury results in decreased contrast sensitivity across spatial frequencies, especially for second-order modulated patterns (13). Binocular disparity perception is also affected by mTBI (14), in addition to inter-ocular signal propagation (12). That contour perception is also affected by mTBI suggests multiple components of the ventral visual pathway, needed for shape and object analysis, may be affected by mTBI. In contrast, motion perception - putatively subserved by the dorsal visual pathwayis not affected in mTBI patients (67). The emerging pattern from these results is that the ventral visual pathway may be more vulnerable to injury, and more studies are needed to assess this possibility.

\section{Visual Field Biases After TBI}

We speculate that the vertical bias (greater efficiency in the lower visual field) may be related to the importance of this hemifield for shape perception-Schmidtmann et al. (68) have reported that while on orientation discrimination tasks performance is balanced between the upper and lower hemifields, there is a distinct advantage in normal individuals in discrimination of complex shapes in the lower visual field. We build on this finding to suggest that perhaps following mTBI, patients increase efficiency selectively in the lower visual field because of its importance to shape recognition, as a compensatory effort.

The left-right bias is admittedly more difficult to explain, but a clue may lie in the bias already present in the normal controlsinternal noise is significantly lower in the right hemifield. We did find that this bias is eliminated by mTBI. We speculate that this bias may be part of normal visual processing, and its disruption by mTBI may be compensated by a biased increase in efficiency corresponding to our observations. 


\section{Internal Noise and Neural Noise}

The concept of noise utilized here-internal noise, captured as a Gaussian random variable within the Lam model-can be understood as a generalization of multiple sources of neural noise including spike-timing variability, synaptic noise, membrane potential variability, etc (69). Complex circuits of neurons would likely exhibit complex noise properties that are not linearly related to the noise within individual units (70-72).

The concept of internal noise, as measured by the equivalent noise technique $(48,50)$ has been effective at capturing a variety of phenomenon that were previously understood as limited by processing capacity or sensitivity, including contrast sensitivity (47), attention (46), and cortical blindness (73). Previous studies had described the observed changes as a modulation of performance capacity or sensitivity but estimates of internal noise within an equivalent noise framework revealed performance was noise-limited not capacity-limited, highlighting the value of a generalized measure of internal noise in characterization performance changes.

A key component of the LAM is the distinction between internal noise and efficiency-the latter denoting the capacity of the system to utilize all the available information. In the present contour task, efficiency has a simple interpretation: it is an estimate of the capacity of the integrative cortical process to pool orientation signals across the retinotopic map to give rise to a coherent representation of the contour. In this respect, mTBI patients did not differ from controls, suggesting that cortical integration is not affected by putative injury.

\section{Neurophysiological Basis of TBI}

TBI results in an array of changes to the brain physiology, including axonal injury (74-78), neuronal death (79-81), neurotransmitter rebalance (82-84), glial activation (85), vascular changes $(86,87)$, and cortical spreading depression (88-90), amongst other factors. Any of these factors would be expected to affect performance on a complex task such as ours. Thus, it is exceedingly difficult to relate the neurophysiological changes that accompany TBI to any aspect of performance on our task.

Crucially, however, participants performance for integrating information during contour perception is not what was affected by $\mathrm{mTBI}-\mathrm{mTBI}$ seemed to only inject noise in this integration mechanism. Compensatory mechanisms activated after TBI maintained a similar degree of cortical integration, thus keeping neural circuits and networks intact, but at the cost of added noise. This is in contradistinction to the notion that tissue loss after TBI causes capacity loss-we speculate that post-TBI compensation seeks to minimize loss of connectivity and circuitry, and the observed deficits are not due to loss of network interactions, but due to increased noise in those interactions.

We did not select participants following the location where the head was hit, nor did we aim to specifically recruit patients who suffered from torsion, direct hit, or indirect jolt, meaning that our cohort included a wide range of mild TBI type. Because none of our participants had any brain lesion (to the visual system or otherwise), heightened internal noise is a general consequence of mTBI stemming from a diffuse cortical imbalance that cannot possibly be restricted to the visual system. We speculate that other sensory modules would be similarly affected by mTBI, and that the LAM could be adapted to capture an general perception internal noise profile.

One cortical location previously thought to be instrumental in the modulation of visual processing noise, namely the Frontal Eye Field (91), and modulation of FEF activity with non-invasive methods such as transcranial magnetic stimulation or direct current stimulation (92), may serve to modulate internal noise and, coincidentally, modulate attentional control effects as well.

\section{Limitations}

Abnormal integrative noise levels are a hallmark of other clinical populations as well. In the Autism Spectrum Disorder for example, noise has been measured via psychophysical methods (93) as in the present study, and operationalized as intraindividual variability in evoked EEG (94) and fMRI responses (95). Crucially, studies that tie physiological and cognitive measurements together allow for stronger claims and more encompassing interpretations, as in the case of schizophrenia (96). As such, functional imaging data should build on our findings to uncover the neural correlates of visual representation internal noise. We found no effect of gender on any of our measurements, but our sample did exhibit a gender bias, and it will be important to include gender as a factor in future mTBI studies because TBI may have gender-specific effects (97).

Increased integration noise was not previously considered as an encompassing feature of mTBI. We therefore stress the value of this encouraging first step toward understanding the functional mechanisms behind visual dysfunctions that follow mild Traumatic Brain Injury.

\section{CONCLUSION}

In conclusion, we have demonstrated that cortical integration following mTBI is limited by abnormally high levels of internal noise as measured by our contour integration task, and that efficiency levels are not altered except in terms of visual field biases, possibly as a compensatory mechanism.

\section{ETHICS STATEMENT}

All participants gave their informed written consent prior to taking part in the experiment. All procedures were in accordance with the Code of Ethics of the World Medical Association (Declaration of Helsinki) and were approved by the Research Ethics Board of the McGill University Health Center.

\section{AUTHOR CONTRIBUTIONS}

TR screened and tested participants, ran statistical analysis, and contributed to writing the manuscript. RF designed the project and contributed to editing the manuscript. $\mathrm{AB}$ designed the experiment and contributed to the statistical analysis and manuscript review. DS contributed to data collection and experiment design. $\mathrm{RH}$ co-designed the project and contributed to manuscript review. 


\section{FUNDING}

This research was funded by the Research Institute of the McGill University Health Center grant and by the Psychological Health and Traumatic Brain Injury Research Program of the U.S. Department of Defense under award W81XWH14-1-0320. Views and opinions of, and endorsements by the author(s) do not reflect those of the US Army or

\section{REFERENCES}

1. Lye TC, Shores EA. Traumatic brain injury as a risk factor for Alzheimer's disease: a review. Neuropsychol. Rev. (2000) 10:115-29. doi: 10.1023/A:1009068804787

2. Millis SR, Rosenthal M, Novack TA, Sherer M, Nick TG, Kreutzer JS, et al. Long-term neuropsychological outcome after traumatic brain injury. J Head Trauma Rehabil. (2001) 16:343-55. doi: 10.1097/00001199-200108000-00005

3. Malojcic B, Mubrin Z, Coric B, Susnic M, Spilich GJ. Consequences of mild traumatic brain injury on information processing assessed with attention and short-term memory tasks. J Neurotrauma. (2008) 25:30-7. doi: 10.1089/neu.2007.0384

4. Masel BE, DeWitt DS. Traumatic brain injury: a disease process, not an event. J Neurotrauma. (2010) 27:1529-40. doi: 10.1089/neu.2010.1358

5. Corrigan JD, Hammond FM. Traumatic brain injury as a chronic health condition. Arch Phys Med Rehabil. (2013) 94:1199-201. doi: 10.1016/j.apmr.2013.01.023

6. Rosenfeld JV, McFarlane AC, Bragge P, Armonda RA, Grimes JB, Ling GS. Blast-related traumatic brain injury. Lancet Neurol. (2013) 12:882-93. doi: 10.1016/S1474-4422(13)70161-3

7. Kandel ER. Principles of Neural Science. 5th ed. New York, NY: McGraw-Hill Education (2013).

8. Yantis S. Sensation and Perception. Basingstoke, UK: Palgrave Macmillan (2013).

9. Adams E. Visual Problems in Traumatic Brain Injury: A Systematic Review of Sequelae and Interventions for the Veteran Population. Vision TBI Final Report. Department of Veterans Affairs, Washington, DC (2009).

10. Kurylo DD, Waxman R, Kezin O. Spatial-temporal characteristics of perceptual organization following acquired brain injury. Brain Injury. (2006) 20:237-44. doi: 10.1080/02699050500487415

11. Chang TT-L, Ciuffreda KJ, Kapoor N. Critical flicker frequency and related symptoms in mild traumatic brain injury. Brain Injury. (2007) 21:1055-62. doi: 10.1080/02699050701591437

12. Spiegel DP, Lague-Beauvais M, Sharma G, Farivar R. Inter-hemispheric wave propagation failures in traumatic brain injury are indicative of callosal damage. Vision Res. (2015) 109:38-44. doi: 10.1016/j.visres.2015. 02.020

13. Spiegel DP, Reynaud A, Ruiz T, Lague-Beauvais M, Hess R, Farivar R. First- and second-order contrast sensitivity functions reveal disrupted visual processing following mild traumatic brain injury. Vision Res. (2016) 122:4350. doi: 10.1016/j.visres.2016.03.004

14. Schmidtmann G, Ruiz T, Reynaud A, Spiegel DP, Laguë-Beauvais M, Hess $\mathrm{RF}$, et al. Sensitivity to binocular disparity is reduced by mild traumatic brain injurydisparity sensitivity after mild traumatic brain injury. Invest Ophthalmol Visual Sci. (2017) 58:2630-5. doi: 10.1167/iovs.17-21845

15. Naghavi HR, Nyberg L. Common fronto-parietal activity in attention, memory, and consciousness: shared demands on integration? Conscious Cogn. (2005) 14:390-425. doi: 10.1016/j.concog.2004.10.003

16. Schlichting ML, Preston AR. Memory integration: neural mechanisms and implications for behavior. Curr Opin Behav Sci. (2015) 1:1-8. doi: 10.1016/j.cobeha.2014.07.005

17. Zhang W, van Ast VA, Klumpers F, Roelofs K, Hermans EJ. Memory contextualization: the role of prefrontal cortex in functional integration across item and context representational regions. J Cogn Neurosci. (2017) 30: 579-93. doi: 10.1162/jocn_a_01218 the Department of Defense. Merit Scholarship Program for Foreign Students (PBEEE-W), Fonds de recherche Nature et technologies, Quebec.

\section{ACKNOWLEDGMENTS}

Research Institute of the McGill University Health Center and US Department of Defense.

18. Field DJ, Hayes A, Hess RF. Contour integration by the human visual system: evidence for a local "association field." Vision Res. (1993) 33:173-93. doi: 10.1016/0042-6989(93)90156-Q

19. Hess RF, Hayes A, Field DJ. Contour integration and cortical processing. J Physiol Paris. (2003) 97:105-19. doi: 10.1016/j.jphysparis.2003.09.013

20. Gilad A, Meirovithz E, Slovin H. Population responses to contour integration: early encoding of discrete elements and late perceptual grouping. Neuron. (2013) 78:389-402. doi: 10.1016/j.neuron.2013.02.013

21. Baldwin AS, Fu M, Farivar R, Hess RF. The equivalent internal orientation and position noise for contour integration. Sci Rep. (2017) 7:13048 doi: 10.1038/s41598-017-13244-Z

22. Pavlovskaya M, Groswasser Z, Keren O, Mordvinov E, Hochstein S Hemispheric visual atentional imbalance in patients with traumatic brain injury. Brain Cogn. (2007) 64:21-9. doi: 10.1016/j.bandc.2006.10.003

23. Giovagnoli AR, Del Pesce M, Mascheroni S, Simoncelli M, Laiacona M, Capitani E. Trail making test: normative values from 287 normal adult controls. Ital J Neurol Sci. (1996) 17:305-9. doi: 10.1007/BF01997792

24. Gauthier L, Dehaut F, Joanette Y. The bells test: a quantitative and qualitative test for visual neglect. Int J Clin Neuropsychol. (1989) 11:49-54. doi: 10.1037/t28075-000

25. Ishiai S, Sugishita M, Ichikawa T, Gono S, Watabiki S. Clockdrawing test and unilateral spatial neglect. Neurology. (1993) 43:106. doi: 10.1212/WNL.43.1_Part_1.106

26. Brainard DH, Vision S. The psychophysics toolbox. Spatial Vision. (1997) 10:433-6. doi: 10.1163/156856897X00357

27. Baldwin AS. alexsbaldwin/MatlabStaircase: v0.9.0 (Version v0.9.0). Zenodo (2019). doi: 10.5281/zenodo.3266142

28. Meese TS. Spatially extensive summation of contrast energy is revealed by contrast detection of micro-pattern textures. J Vis. (2010) 10:14. doi: $10.1167 / 10.8 .14$

29. Wertheimer M. Laws of organization in perceptual forms. In: Ellis W, editor. A Source Book of Gestalt Psychology. London: Routledge and Kegan Paul (1938). p. 71-88. doi: 10.1037/11496-005

30. Jäkel F, Wichmann FA. Spatial four-alternative forced-choice method is the preferred psychophysical method for naïve observers. J Vision. (2006) 6:130722. doi: $10.1167 / 6.11 .13$

31. Noguchi K, Gel YR, Brunner E, Konietschke F. nparLD: an R software package for the nonparametric analysis of longitudinal data in factorial experiments. $J$ Stat Softw. (2012) 50:5. doi: 10.18637/jss.v050.i12

32. R Core Team (2013). R: A Language and Environment for Statistical Computing. Vienna: R Foundation for Statistical Computing.

33. Prins N, Kingdom FAA. Applying the model-comparison approach to test specific research hypotheses in psychophysical research using the palamedes toolbox. Front Psychol. (2018) 9:1250. doi: 10.3389/fpsyg.2018 01250

34. North D. The absolute sensitivity of radio receivers. RCA Rev. (1942) 6:33243.

35. Friis HT. Noise figures of radio receivers. Proc. IRE. (1944) 32:419-22. doi: 10.1109/JRPROC.1944.232049

36. Mumford WW, Scheibe EH. Noise Performance Factors in Communication Systems. Norwood, OH: Horizon House Publications (1968).

37. Pelli DG. Effects of Visual Noise (Ph.D. dissertation). University of Cambridge, Cambridge, United Kingdom (1981).

38. Baldwin AS, Baker DH, Hess RF. What do contrast threshold equivalent noise studies actually measure? Noise vs. nonlinearity 
in different masking paradigms. PLoS ONE. (2016) 11:e0150942. doi: 10.1371/journal.pone.0150942

39. Tukey JW. Schematic plots. In: Exploratory Data Analysis. Reading, MA: Addison Wesley Publishing Company (1970). p. 47-8.

40. Hoaglin DC, Mosteller F, Tukey JW. Boxplots and batch comparison. In: Hoaglin DC, Mosteller F, Tukey JW, editors. Understanding Robust and Exploratory Data Analysis. New York, NY: Wiley (1983). p. 58-96.

41. Bryant RA, Harvey AG. Postconcussive symptoms and posttraumatic stress disorder after mild traumatic brain injury. J Nervous Ment Dis. (1999) 187:302-5. doi: 10.1097/00005053-199905000-00006

42. Emanuelson I, Andersson Holmkvist E, Björklund R, Stålhammar D. Quality of life and post-concussion symptoms in adults after mild traumatic brain injury: a population-based study in western Sweden. Acta Neurol Scand. (2003) 108:332-8. doi: 10.1034/j.1600-0404.2003.00155.x

43. Sigurdardottir S, Andelic N, Roe C, Jerstad T, Schanke A-K. Postconcussion symptoms after traumatic brain injury at 3 and 12 months post-injury: a prospective study. Brain Injury. (2009) 23:489-97. doi: 10.1080/02699050902926309

44. Fagerholm ED, Hellyer PJ, Scott G, Leech R, Sharp DJ. Disconnection of network hubs and cognitive impairment after traumatic brain injury. Brain. (2015) 138:1696-709. doi: 10.1093/brain/awv075

45. Gilad A, Meirovithz E, Leshem A, Arieli A, Slovin H. Collinear stimuli induce local and cross-areal coherence in the visual cortex of behaving monkeys. PLoS ONE. (2012) 7:e49391. doi: 10.1371/journal.pone.0049391

46. Lu ZL, Dosher BA. External noise distinguishes attention mechanisms. Vision Res. (1998) 38:1183-98. doi: 10.1016/S0042-6989(97)00273-3

47. Skoczenski AM, Norcia AM. Neural noise limitations on infant visual sensitivity. Nature. (1998) 391:697-700. doi: 10.1038/35630

48. Lu ZL, Dosher BA. Characterizing human perceptual inefficiencies with equivalent internal noise. J Optic Soc Am A. (1999) 16:764-78. doi: 10.1364/JOSAA.16.000764

49. Pelli DG, Farell B. Why use noise? J Opt Soc Am A. (1999) 16:647-53. doi: 10.1364/JOSAA.16.000647

50. Lu ZL, Dosher BA. Characterizing observers using external noise and observer models: assessing internal representations with external noise. Psychol Rev. (2008) 115:44-82. doi: 10.1037/0033-295X.115.1.44

51. Ware JB, Hart T, Whyte J, Rabinowitz A, Detre JA, Kim J. Inter-subject variability of axonal injury in diffuse traumatic brain injury. J Neurotrauma. (2017) 34:2243-53. doi: 10.1089/neu.2016.4817

52. Yue JK, Robinson CK, Winkler EA, Upadhyayula PS, Burke JF, Pirracchio $\mathrm{R}$, et al. Circadian variability of the initial Glasgow Coma Scale score in traumatic brain injury patients. Neurobiol Sleep Circadian Rhyth. (2017) 2:85-93. doi: 10.1016/j.nbscr.2016.09.002

53. Pasupathy A, Connor CE. Responses to contour features in macaque area V4. J Neurophysiol. (1999) 82:2490-502. doi: 10.1152/jn.1999.82.5.2490

54. Kisvárday ZF, Toth E, Rausch M, Eysel UT. Orientation-specific relationship between populations of excitatory and inhibitory lateral connections in the visual cortex of the cat. Cereb Cortex. (1997) 7:605-18. doi: 10.1093/cercor/7.7.605

55. Field DJ, Hayes A. Contour integration and the lateral connections of V1 neurons. In: Chalupa LM, Werner JS, editors. The Visual Neurosciences. Cambridge, MA: The MIT Press (2004). p. 1069-79.

56. Elder JH, Goldberg RM. Ecological statistics of Gestalt laws for the perceptual organization of contours. J Vis. (2002) 2:324-53. doi: 10.1167/2.4.5

57. Koffka K. Principles of Gestalt Psychology. Abingdon, UK: Routledge (2013). doi: 10.4324/9781315009292

58. Zipser K, Lamme VA, Schiller PH. Contextual modulation in primary visual cortex. $J$ Neurosci. (1996) 16:7376-89. doi: 10.1523/JNEUROSCI.16-22-07376.1996

59. Lamme VA, Super H, Spekreijse H. Feedforward, horizontal, and feedback processing in the visual cortex. Curr Opin Neurobiol. (1998) 8:529-35. doi: 10.1016/S0959-4388(98)80042-1

60. May KA, Hess RF. Effects of element separation and carrier wavelength on detection of snakes and ladders: implications for models of contour integration. J Vis. (2008) 8:1-23. doi: 10.1167/8.13.4

61. Hansen BC, May KA, Hess RF. One "shape" fits all: the orientation bandwidth of contour integration. J Vis. (2014) 14:17. doi: 10.1167/14.13.17
62. Blakemore C, Tobin EA. Lateral inhibition between orientation detectors in the cat's visual cortex. Exp Brain Res. (1972) 15:439-40. doi: 10.1007/BF00234129

63. von der Heydt R, Peterhans E. Mechanisms of contour perception in monkey visual cortex. I. Lines of pattern discontinuity. J Neurosci. (1989) 9:1731-48. doi: 10.1523/JNEUROSCI.09-05-01731.1989

64. Knierim JJ, Van Essen DC. Neuronal responses to static texture patterns in area V1 of the alert macaque monkey. J Neurophysiol. (1992) 67:961-80. doi: $10.1152 /$ jn.1992.67.4.961

65. Sillito AM, Grieve KL, Jones HE, Cudeiro J, Davis J. Visual cortical mechanisms detecting focal orientation discontinuities. Nature. (1995) 378:492-6. doi: 10.1038/378492a0

66. Lachapelle J, Ouimet C, Bach M, Ptito A, McKerral M. Texture segregation in traumatic brain injury-a VEP study. Vis Res. (2004) 44:2835-42. doi: 10.1016/j.visres.2004.06.007

67. Costa TL, Zaninotto ALC, Benute GG, De Lúcia MCS, Paiva WS, Wagemans J, et al. Perceptual organization deficits in traumatic brain injury patients. Neuropsychologia. (2015) 78:142-52. doi: 10.1016/j.neuropsychologia.2015.10.008

68. Schmidtmann G, Logan AJ, Kennedy GJ, Gordon GE, Loffler G. Distinct lower visual field preference for object shape. J Vis. (2015) 15:18. doi: 10.1167/15.5.18

69. Faisal AA, Selen LPJ, Wolpert DM. Noise in the nervous system. Nat Rev Neurosci. (2008) 9:292-303. doi: 10.1038/nrn2258

70. Laughlin SB, Sejnowski TJ. Communication in neuronal networks. Science. (2003) 301:1870-4. doi: 10.1126/science.1089662

71. Prinz AA, Bucher D, Marder E. Similar network activity from disparate circuit parameters. Nature neuroscience. (2004) 7:1345-52. doi: 10.1038/nn1352

72. Faisal AA, White JA, Laughlin SB. Ion-channel noise places limits on the miniaturization of the brain's wiring. Curr Biol. (2005) 15:1143-9. doi: 10.1016/j.cub.2005.05.056

73. Cavanaugh MR, Zhang R, Melnick MD, Das A, Roberts M, Tadin D, et al. Visual recovery in cortical blindness is limited by high internal noise. J Vis. (2015) 15:9. doi: 10.1167/15.10.9

74. Povlishock J, Becker D, Cheng C, Vaughan G. Axonal change in minor head injury. J Neuropathol Exp Neurol. (1983) 42:225-42. doi: 10.1097/00005072-198305000-00002

75. Hammoud DA, Wasserman BA. Diffuse axonal injuries: pathophysiology and imaging. Neuroimaging Clin. (2002) 12:205-16. doi: 10.1016/S1052-5149(02)00011-4

76. Rubovitch V, Ten-Bosch M, Zohar O, Harrison CR, Tempel-Brami C, Stein E, et al. A mouse model of blast-induced mild traumatic brain injury. Exp Neurol. (2011) 232:280-9. doi: 10.1016/j.expneurol.2011.09.018

77. Sharp DJ, Ham TE. Investigating white matter injury after mild traumatic brain injury. Curr Opin Neurol. (2011) 24:558-63. doi: 10.1097/WCO.0b013e32834cd523

78. Johnson VE, Stewart W, Smith DH. Axonal pathology in traumatic brain injury. Exp Neurol. (2013) 246:35-43. doi: 10.1016/j.expneurol.2012.01.013

79. Ross DT, Graham DI, Adams JH. Selective loss of neurons from the thalamic reticular nucleus following severe human head injury. J Neurotrauma. (1993) 10:151-65. doi: 10.1089/neu.1993.10.151

80. Rink A, Fung KM, Trojanowski JQ, Lee VM, Neugebauer E, McIntosh TK. Evidence of apoptotic cell death after experimental traumatic brain injury in the rat. Am J Pathol. (1995) 147:1575-83.

81. Raghupathi R. Cell death mechanisms following traumatic brain injury. Brain Pathol. (2004) 14:215-22. doi: 10.1111/j.1750-3639.2004.tb00056.x

82. Walter B, Brust P, Fuchtner F, Muller M, Hinz R, Kuwabara H, et al. Agedependent effects of severe traumatic brain injury on cerebral dopaminergic activity in newborn and juvenile pigs. J Neurotrauma. (2004) 21:1076-89. doi: 10.1089/0897715041651024

83. Hunt RF, Scheff SW, Smith BN. Synaptic reorganization of inhibitory hilar interneuron circuitry after traumatic brain injury in mice. J Neurosci. (2011) 31:6880-90. doi: 10.1523/JNEUROSCI.0032-11.2011

84. Bigler ED. Systems biology, neuroimaging, neuropsychology, neuroconnectivity and traumatic brain injury. Front Syst Neurosci. (2016) 10:55. doi: 10.3389/fnsys.2016.00055

85. Ramlackhansingh AF, Brooks DJ, Greenwood RJ, Bose SK, Turkheimer FE, Kinnunen KM, et al. Inflammation after trauma: microglial activation and 
traumatic brain injury. Ann Neurol. (2011) 70:374-83. doi: 10.1002/ana. 22455

86. Bonne O, Gilboa A, Louzoun Y, Kempf-Sherf O, Katz M, Fishman Y, et al. Cerebral blood flow in chronic symptomatic mild traumatic brain injury. Psychiatry Res. (2003) 124:141-52. doi: 10.1016/S0925-4927(03)00109-4

87. Wang Y, West JD, Bailey JN, Westfall DR, Xiao H, Arnold TW, et al. Decreased cerebral blood flow in chronic pediatric mild TBI: an MRI perfusion study. Dev Neuropsychol. (2015) 40:40-4. doi: 10.1080/87565641.2014.979927

88. Giza CC, Hovda DA. The neurometabolic cascade of concussion. J Athletic Train. (2001) 36:228. doi: 10.1016/S0008-4182(01)80051-X

89. Church AJ, Andrew RD. Spreading depression expands traumatic injury in neocortical brain slices. J Neurotrauma. (2005) 22:277-90. doi: 10.1089/neu.2005.22.277

90. Lauritzen M, Dreier JP, Fabricius M, Hartings JA, Graf R, Strong AJ. Clinical relevance of cortical spreading depression in neurological disorders: migraine, malignant stroke, subarachnoid and intracranial hemorrhage, and traumatic brain injury. J Cereb Blood Flow Metab. (2011) 31:17-35. doi: $10.1038 /$ jcbfm.2010.191

91. Noudoost B, Moore T. Control of visual cortical signals by prefrontal dopamine. Nature. (2011) 474:372-5. doi: 10.1038/nature09995

92. Grosbras MH, Paus T. Transcranial magnetic stimulation of the human frontal eye field facilitates visual awareness. Eur J Neurosci. (2003) 18:3121-6. doi: 10.1111/j.1460-9568.2003.03055.x

93. Vilidaite G, Yu M, Baker DH. Internal noise estimates correlate with autistic traits. Autism Res. (2017) 10:1384-91. doi: 10.1002/aur.1781
94. Milne E. Increased intra-participant variability in children with autistic spectrum disorders: evidence from single-trial analysis of evoked EEG. Front Psychol. (2011) 2:51. doi: 10.3389/fpsyg.2011.00051

95. Haigh SM, Heeger DJ, Dinstein I, Minshew N, Behrmann M. Cortical variability in the sensory-evoked response in autism. J Autism Dev Disord. (2015) 45:1176-90. doi: 10.1007/s10803-014-2276-6

96. Winterer G, Coppola R, Goldberg TE, Egan MF, Jones DW, Sanchez $\mathrm{CE}$, et al. Prefrontal broadband noise, working memory, and genetic risk for schizophrenia. Am J Psychiatry. (2004) 161:490-500. doi: 10.1176/appi.ajp.161.3.490

97. Farace E, Alves WM. Do women fare worse: a metaanalysis of gender differences in traumatic brain injury outcome. J Neurosurg. (2000) 93:539-45. doi: $10.3171 /$ jns.2000.93.4.0539

Conflict of Interest Statement: The authors declare that the research was conducted in the absence of any commercial or financial relationships that could be construed as a potential conflict of interest.

Copyright (C) 2019 Ruiz, Baldwin, Spiegel, Hess and Farivar. This is an open-access article distributed under the terms of the Creative Commons Attribution License (CC $B Y)$. The use, distribution or reproduction in other forums is permitted, provided the original author(s) and the copyright owner(s) are credited and that the original publication in this journal is cited, in accordance with accepted academic practice. No use, distribution or reproduction is permitted which does not comply with these terms. 